\title{
Constructing Highly Incident Configurations
}

\author{
Leah Wrenn Berman
}

Received: 6 April 2010 / Revised: 20 July 2010 / Accepted: 26 July 2010 /

Published online: 18 August 2010

(C) Springer Science+Business Media, LLC 2010

\begin{abstract}
A geometric $(q, k)$-configuration is a collection of points and straight lines in the Euclidean plane in which each point lies on $q$ lines and each line passes through $k$ points. We say a $(q, k)$-configuration is highly incident when one (or both) of $q$ or $k$ is strictly greater than 4 . In this paper, two simple lemmas are used to construct infinite classes of $(2 q, 2 k)$-configurations for any $q, k \geq 2$; the resulting configurations have non-trivial dihedral symmetry. In particular, this construction produces the only known infinite class of symmetric 6-configurations.
\end{abstract}

Keywords Configurations $\cdot$ Incidence geometry $\cdot$ Symmetry

A geometric $(q, k)$-configuration is a collection of points and straight lines in the Euclidean plane in which every point lies on $q$ lines and every line passes through $k$ points. If the number of points $p$ and the number of lines $n$ is relevant, we refer to a $\left(p_{q}, n_{k}\right)$ configuration. If $q=k$, we simply refer to a $k$-configuration. We say a $(q, k)$-configuration is symmetric if, under rotations and reflections of the plane mapping the configuration to itself, there are fewer symmetry classes of points than the number of points in the configuration, and similarly for lines: configurations that are highly symmetric have a small number of symmetry classes of points and lines. (Note that this use of the word "symmetric" is different from its use in, e.g., [8]. In this and other references, a symmetric configuration refers to a $\left(p_{q}, n_{k}\right)$ configuration in which $q=k$ (and, by counting incidences, $p=n$ ), probably related to the notion of a symmetric balanced incomplete block design (BIBD), since combinatorially, a $\left(p_{q}, n_{k}\right)$ configuration is a $(p, n, q, k, 1)$-BIBD. Grünbaum has an amusing discussion of this terminological collision in $[14$, p. 16], where he proposes using the term

L.W. Berman $(\bowtie)$

Department of Mathematics \& Statistics, University of Alaska Fairbanks, P.O. Box 756660,

Fairbanks, AK, USA

e-mail: 1wberman@alaska.edu 
balanced for $k$-configurations and reserving the term symmetric for configurations that exhibit geometric symmetry.)

Geometric 3-configurations have been studied since the late 1800s. Geometric 4-configurations have been studied fairly extensively for the past 20 years, beginning with the first known publication of an intelligible diagram of a 4-configuration, in [15], and continuing in various articles, e.g., [1, 3, 9-12]. However, there has been very little investigation into $k$-configurations with $k>4$; there have been a few articles discussing 5-configurations [5, 6] and a very short section in [14, Sect. 4.1], but only negative results for the existence of particularly highly symmetric 6-configurations (in particular, that there are no astral 6-configurations, which would have three symmetry classes of points and lines) [2]. There have been basically no positive results concerning the existence of $(q, k)$ - or $k$-configurations which have any reasonable symmetry for large $q, k$ (e.g., greater than or equal to 6; see [14, pp. 239-242] for a summary of the few known results). In this paper, we describe a construction method for producing, for any $m>2(q+k-1)$, a family of $(2 q, 2 k)$ configurations for any $q, k \geq 2$ which have the dihedral symmetry of the $m$-gon.

We say a configuration is highly incident if there are more than four points incident with every line, or if there are more than four lines passing through every point. The current state of research on highly incident configurations is discussed in detail (as much as is known, that is) in Sects. 4.1-4.5 of Branko Grünbaum's recent monograph on configurations [14]. Briefly, there are five connected astral $(4,6)$ - and $(6,4)$-configurations which are known, plus configurations formed by taking evenly spaced disconnected copies of those [2]. There are a few $(5,4)-$ and $(7,4)$-configurations formed by adding appropriate diameters to less highly incident configurations $[2,4,13]$. There are some $(4,5)$ - and $(5,4)$-configurations formed by "mixing" 4-configurations [4]. Finally, there are some highly incident "floral" configurations [7, 14]; however, these have the property that the symmetry of the entire configuration is low compared to the total number of points and lines of the configuration. For example, Fig. 4.5.17 of [14, Sect. 4.5] shows a $\left(250_{4}, 125_{8}\right)$ floral configuration with symmetry group $d_{5}$. In contrast, the smallest $(4,8)$-configuration which may be produced by the method outlined in this paper is a $\left(110_{4}, 55_{8}\right)$ configuration with $\left(\begin{array}{l}5 \\ 3\end{array}\right)=10$ symmetry classes of points, $\left(\begin{array}{l}5 \\ 4\end{array}\right)=5$ symmetry classes of lines, and symmetry group $d_{11}$, shown in Fig. 1 .

\section{Constructing Symmetry Classes of Points and Lines}

To construct highly incident configurations, we will begin with a regular convex $m$ gon and add various symmetry classes of points and lines in a systematic way.

Some preliminary notation is required. If $P$ and $Q$ are points, the line containing $P$ and $Q$ is denoted $P \vee Q$. If $l$ and $p$ are lines, the intersection of $l$ and $p$ is denoted $l \wedge p$. Throughout the paper, all indices are taken modulo $m$. If $w_{0}, w_{1}, \ldots, w_{m-1}$ are the vertices of a regular convex $m$-gon, a line of span $s$ is any line of the form $w_{i} \vee w_{i+s}$; similarly, if $l_{0}, l_{1}, \ldots, l_{m-1}$ are lines which form the edges of a regular convex $m$-gon, the $t$ th intersection of the lines $l$ is any point of the form $l_{i} \wedge l_{i-t}$. Moreover, note that if $w_{i}=l_{i} \wedge l_{i-t}$, then $l_{i}=w_{i} \vee w_{i+t}$; that is, if a set of vertices is 


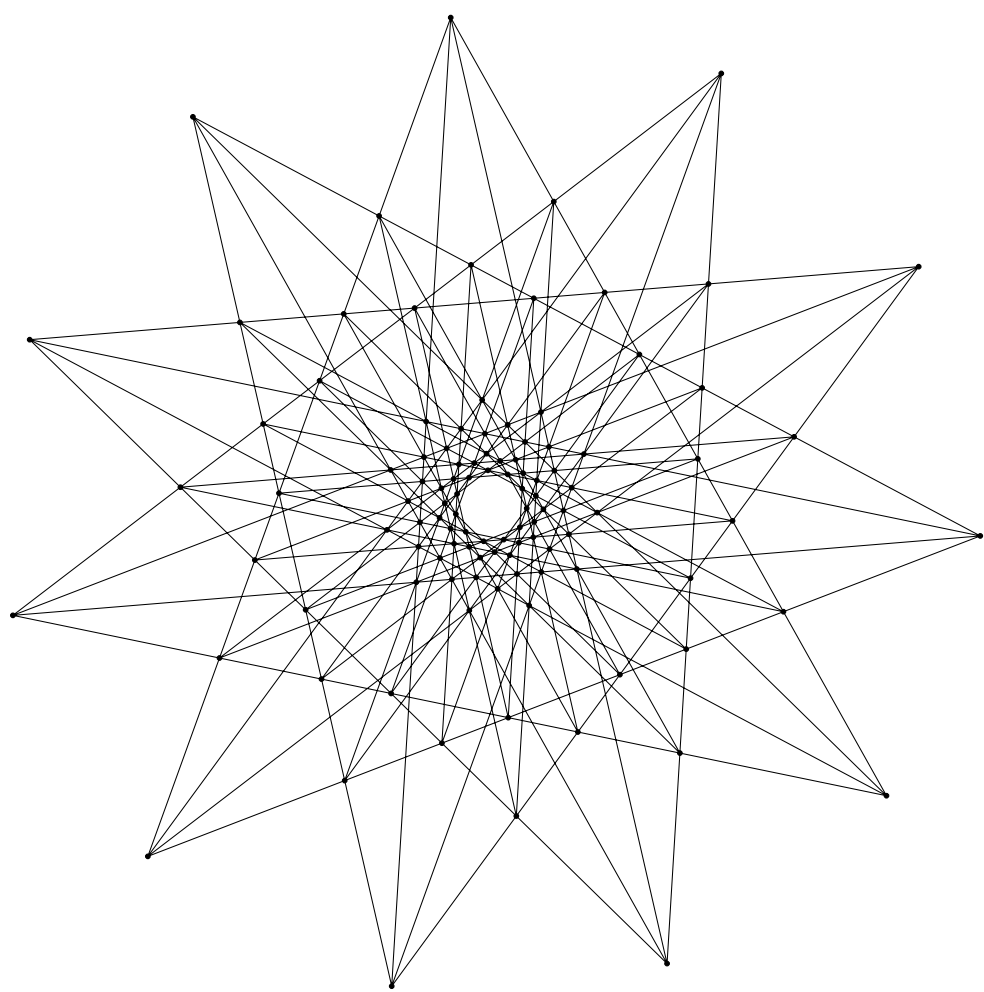

Fig. 1 A highly symmetric $(4,8)$-configuration; in particular, this is a $\left(110_{4}, 55_{8}\right)$ configuration, with the symmetries of an 11-gon

formed as the $t$ th intersection points of a set of lines, then from the viewpoint of the vertices, the lines are of span $t$. See Fig. 2 for an illustration.

To construct additional sets of points and lines with appropriate incidences, we will use the following lemmas, illustrated in Figs. 3 and 4.

Theorem 1 (Crossing Spans Lemma) Given a regular $m$-gon $\mathcal{M}$ with vertices $u_{0}, u_{1}, \ldots, u_{m-1}$ and diagonals $\Theta_{i}=u_{i} \vee u_{i+\alpha}$ of span $\alpha$ and $\Psi_{i}=u_{i} \vee u_{i+\beta}$ of span $\beta$, suppose that $x_{0}=(1-\lambda) u_{0}+\lambda u_{\alpha}$ is an arbitrary point on $\Theta_{0}$, and construct other points $x_{i}$ to be the rotations of $x_{0}$ through $\frac{2 \pi i}{m}\left(\right.$ so that $\left.x_{i}=(1-\lambda) u_{i}+\lambda u_{i+\alpha}\right)$, forming a second regular, convex $m$-gon $\mathcal{N}$. Construct diagonals $\Gamma_{i}$ of span $\beta$ with respect to the $x_{i}$ : that is, let $\Gamma_{i}=x_{i} \vee x_{i+\beta}$. Let $y_{i}=\Gamma_{i} \wedge \Psi_{i}$ and let $y_{i}^{\prime}=$ $\Gamma_{i-\alpha} \wedge \Psi_{i}$.Then $y_{i}=y_{i}^{\prime}$.

This lemma was proved with slightly different notation in [3] and was stated with the current notation in [6].

Corollary 2 (Points Completion Lemma (PCL)) If, in the context of the Crossing Spans Lemma the points $x_{i}$ are chosen to be $x_{i}=\Theta_{i} \wedge \Theta_{i-\delta}$, then the points $y_{i}$ also lie on $\Psi_{i-\delta}$. 
Fig. 2 If $w_{i}=l_{i} \wedge l_{i-t}$, then $l_{i}=w_{i} \vee w_{i+t}$; for example, here $w_{0}=l_{0} \wedge l_{-2}=l_{0} \wedge l_{5}$ and $l_{0}=w_{0} \vee w_{2}$. The point $w_{0}$ and line $l_{0}$ are shown larger

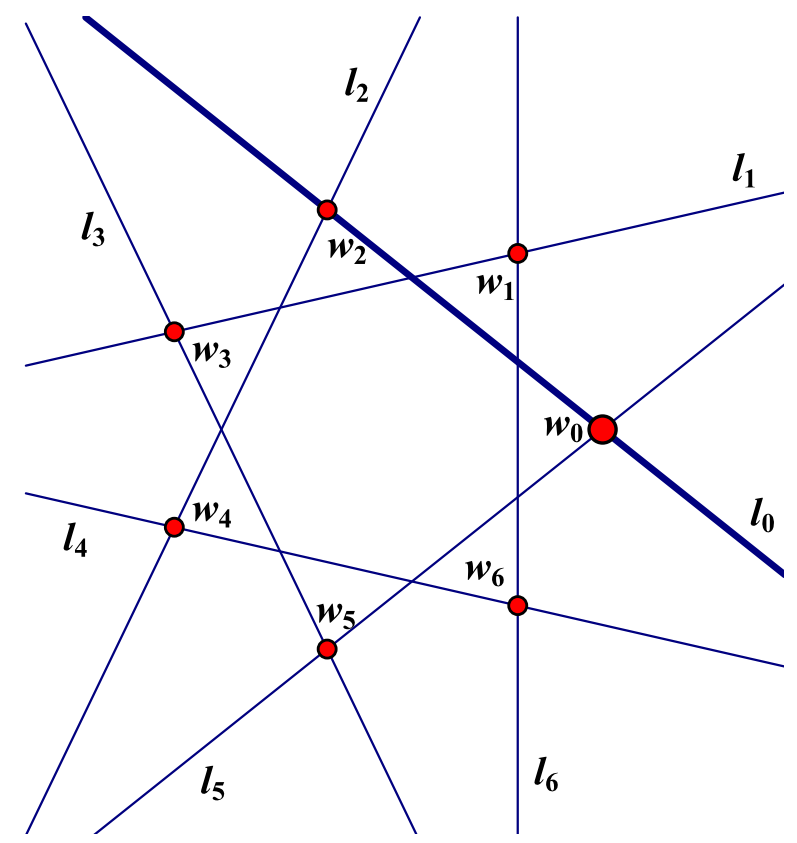

Proof If $x_{i}=\Theta_{i} \wedge \Theta_{i-\delta}$, then $\Theta_{i}=x_{i} \vee x_{i+\delta}$. We can apply the Crossing Spans Lemma twice. For the second application, we let $\alpha^{\prime}=\delta$ and define

$$
\begin{aligned}
u_{i}^{\prime} & =x_{i}, \\
\Theta_{i}^{\prime} & =u_{i}^{\prime} \vee u_{i+\alpha^{\prime}}^{\prime}=x_{i} \vee x_{i+\delta}=\Theta_{i}, \\
x_{i}^{\prime} & =u_{i}, \\
\Psi_{i}^{\prime} & =u_{i}^{\prime} \vee u_{i+\beta}^{\prime}=x_{i} \vee x_{i+\beta}=\Gamma_{i}, \\
\Gamma_{i}^{\prime} & =x_{i}^{\prime} \vee x_{i+\beta}^{\prime}=u_{i} \vee u_{i+\beta}=\Psi_{i}, \\
y_{i}^{\prime} & =\Gamma_{i}^{\prime} \wedge \Psi_{i}^{\prime}=y_{i} .
\end{aligned}
$$

Applying the Crossing Spans Lemma, we conclude that $\Gamma_{i}^{\prime}=\Psi_{i}, \Gamma_{i-\alpha^{\prime}}^{\prime}=\Psi_{i-\delta}$ and $\Psi_{i}^{\prime}=\Gamma_{i}$ are all concurrent at $y_{i}$.

Lemma 3 (Dual Crossing Spans Lemma) Let $U_{0}, \ldots, U_{m-1}$ be the (extended) sides of a regular, convex $m$-gon, and let $\theta_{i}=U_{i} \wedge U_{i+\alpha}$. (Note that consequently, $U_{i}=$ $\theta_{i} \vee \theta_{i-\alpha}$.) Let $X_{0}$ be an arbitrary line passing through $\theta_{0}$, and let $X_{i}$ be the rotation of $X_{0}$ through $\frac{2 \pi i}{m}$. Define $\psi_{i}=U_{i} \wedge U_{i+\beta}$ and $\gamma_{i}=X_{i} \wedge X_{i+\beta}$.

Let $Y_{i}=\gamma_{i} \vee \psi_{i}$ and $Y_{i}^{\prime}=\gamma_{i-\alpha} \vee \psi_{i}$. Then $Y_{i}=Y_{i}^{\prime}$. That is, the points $\psi_{i}, \gamma_{i}$ and $\gamma_{i-\alpha}$ are collinear.

This is the dual formulation of the Crossing Spans Lemma, formed by switching the roles of the points and lines (and switching the case of the variables, for clarity); see Fig. 4a for an illustration. 
Fig. 3 Illustration of the Crossing Spans Lemma and the Point Completion Lemma. In both cases, $m=9$, and $\alpha=2$, $\beta=3, \delta=1$, and the 0th lines of each set are shown thick. In (b), the lines $\Theta_{-\delta}$ and $\Psi_{-\delta}$ are shown thick and dashed

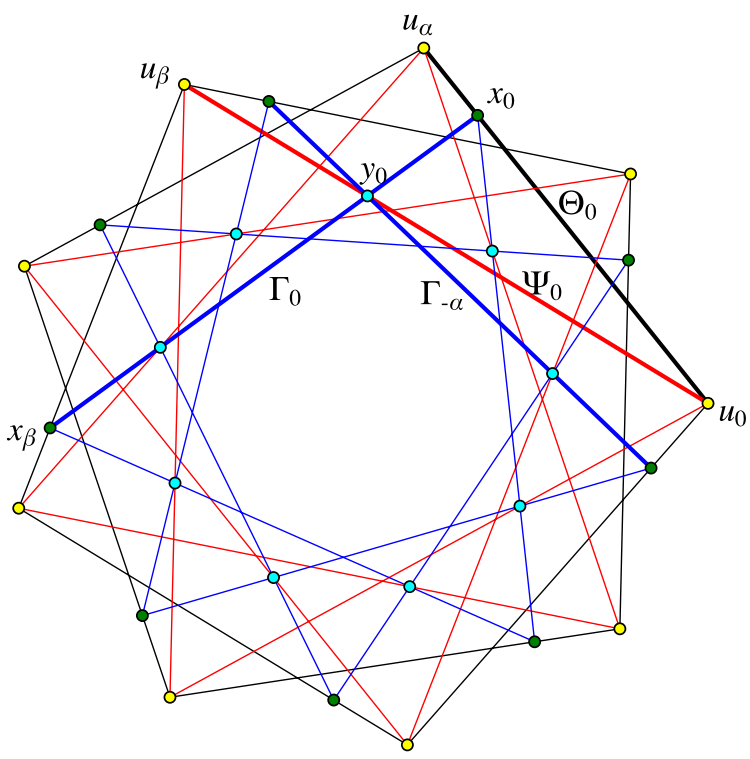

(A) Crossing Spans Lemma.

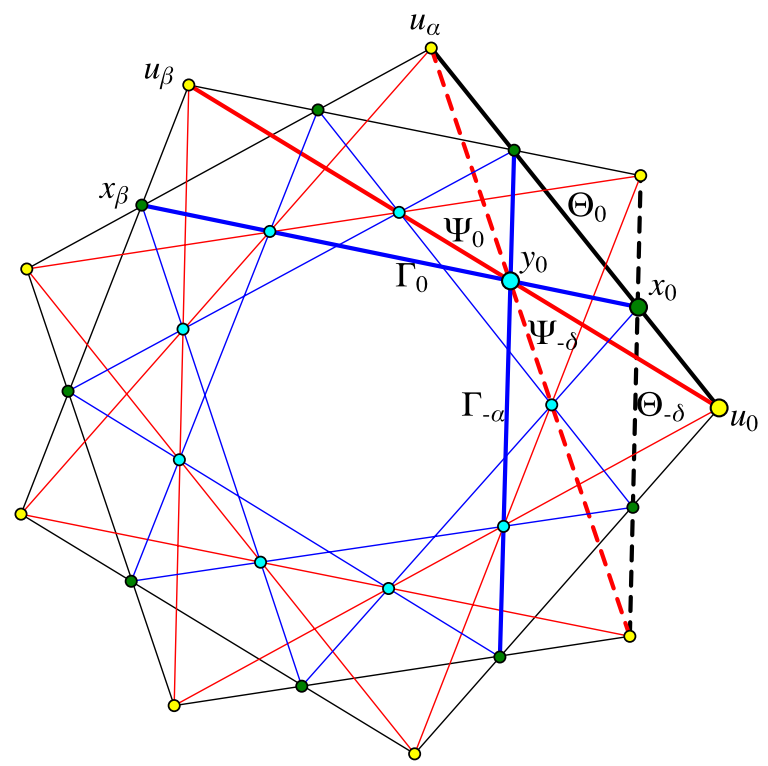

(B) Points Completion Lemma.

Corollary 4 (Lines Completion Lemma (LCL)) In the situation of the Dual Crossing Spans Lemma, if line $X_{0}$ is chosen to pass through $\theta_{\delta}$ (that is, if $\left.X_{i}=\theta_{i} \vee \theta_{i+\delta}\right)$, then point $\psi_{i+\delta}$ also lies on line $Y_{i}$. 
Fig. 4 Illustration of the Dual Crossing Spans Lemma and the Lines Completion Lemma. In both cases, $m=9$, and $\alpha=2, \beta=3, \delta=1$, and the 0th line of each set is shown thick

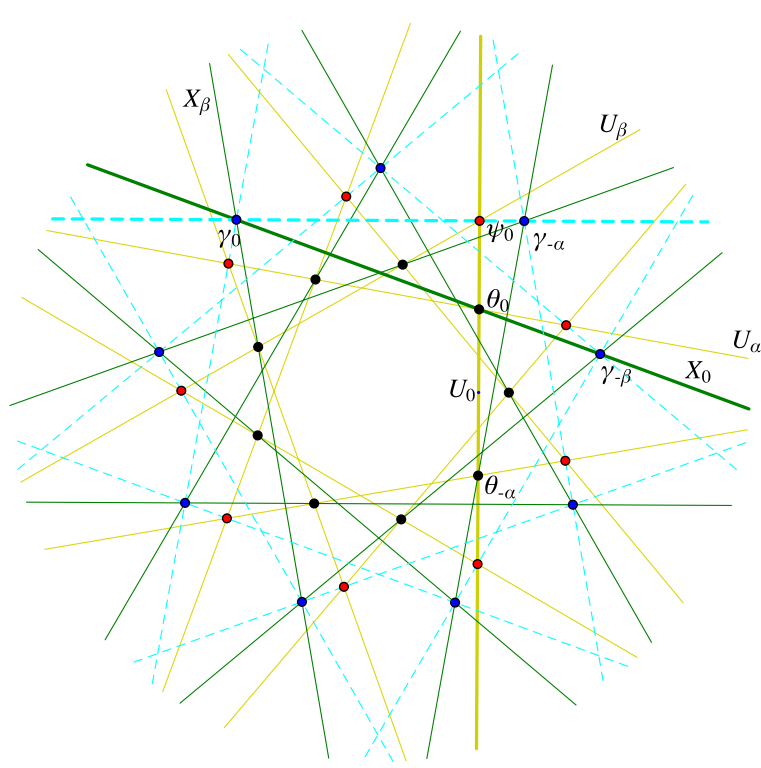

(A) Dual Crossing Spans Lemma.

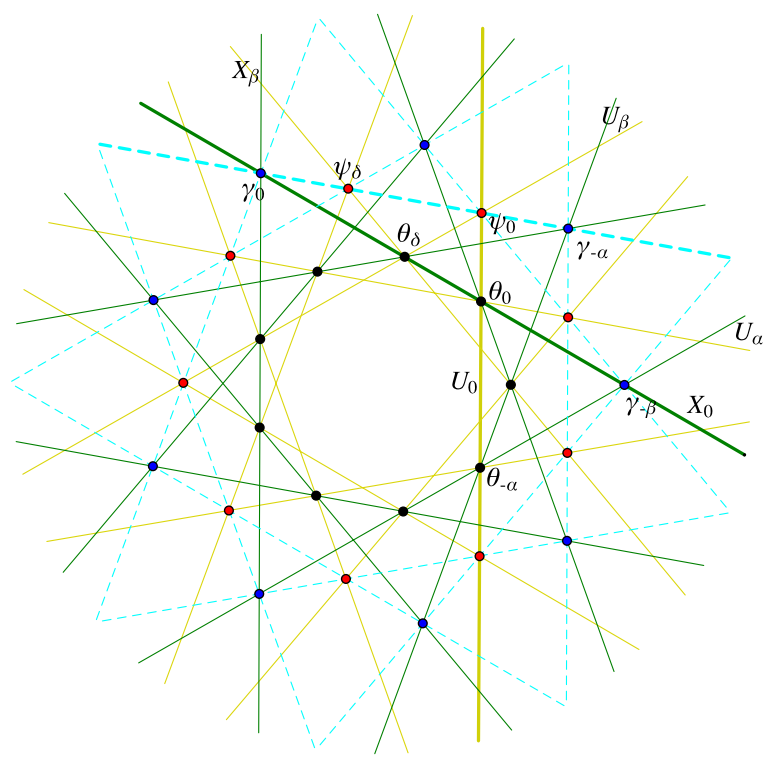

(B) Lines Completion Lemma.

This is the dual formulation of the PCL; see Fig. 4b for an illustration.

Notice that the Points Completion Lemma is equivalent to the existence of the well-understood trivial 3-astral 4-configuration $m \#(\alpha, \delta ; \beta, \alpha ; \delta, \beta)$ and the Lines Completion Lemma to the existence of the polar trivial 3-astral 4-configuration 
$m \#(\delta, \beta ; \alpha, \delta ; \beta, \alpha)$, which have been discussed in detail in other places; Sect. 3.7 in [14] has a good summary and discussion.

To construct highly incident configurations, we will repeatedly apply the Points Completion Lemma and Lines Completion Lemma to the points and lines of an initial incidence structure in order to construct additional symmetry classes of points and lines with the desired incidences.

\section{Constructing Highly Incident Configurations}

Choose integers $q, k \geq 2$ and suppose $m>2(q+k-1)$ and

$$
1 \leq s_{0}, s_{1}, \ldots, s_{q-2}, t_{0}, t_{1}, \ldots, t_{k-1}<\frac{m}{2}
$$

are $q+k-1$ distinct positive integers. Let $S=\{0,1, \ldots, q-2\}$ and $T=$ $\{0,1, \ldots, k-1\}$ be the associated index sets for the sets $\left\{s_{0}, s_{1}, \ldots, s_{q-2}\right\}$ and $\left\{t_{0}, t_{1}, \ldots, t_{k-1}\right\}$ respectively. We define a (combinatorial) configuration with parameter list

$$
\left(\left(s_{0}, s_{1}, \ldots, s_{q-2}\right),\left(t_{0}, t_{1}, \ldots, t_{k-1}\right)\right)
$$

to be a collection of points and lines such that for $i=0,1 \ldots, m-1$, the points $\left(v_{\tau}^{\hat{\sigma}}\right)_{i}$ are indexed by all subsets $\hat{\sigma}$ of $S$ (including the empty subset) and $\tau$ of $T$ in which $|\hat{\sigma}|=|\tau|-1$, and the lines $\left(L_{\tau}^{\sigma}\right)_{i}$ are indexed by all subsets $\sigma$ of $S$ and $\tau$ of $T$ (including the empty subsets) where $|\sigma|=|\tau|$; incidence between points and lines is determined by the condition that a vertex $\left(v_{\tau}^{\hat{\sigma}}\right)_{i}$ is incident with a line $\left(L_{\tau}^{\sigma}\right)_{i}$ when $\hat{\sigma} \subset \sigma$ and $|\hat{\sigma}|+1=|\sigma|=|\tau|$.

The bulk of the paper will be spent discussing the following theorem.

Theorem 5 For any integer $m>2(q+k-1)$, it is possible to construct a geometric $(2 q, 2 k)$-configuration with parameter list

$$
\left(\left(s_{0}, s_{1}, \ldots, s_{q-2}\right),\left(t_{0}, t_{1}, \ldots, t_{k-1}\right)\right)
$$

and symmetry group $d_{m}$.

The resulting configuration has $\left(\begin{array}{c}q+k-1 \\ k-1\end{array}\right)$ symmetry classes of points and $\left(\begin{array}{c}q+k-1 \\ k\end{array}\right)$ symmetry classes of lines when $k \leq q-1$ and $\left(\begin{array}{c}q+k-1 \\ q-1\end{array}\right)$ symmetry classes of points and $\left(\begin{array}{c}q+k-1 \\ q\end{array}\right)$ symmetry classes of lines when $q-1<k$.

To make clear the complex notation of the theorem, the next section will give an overview of the construction method and will carefully apply the construction method to several small cases, in particular to the explicit construction of a 6-configuration with parameter list $((4,5),(3,2,1))$. In Sect. 4 , we will prove that the construction technique is valid and that the incidence structure that corresponds to a parameter list $\left(\left(s_{0}, \ldots, s_{q-2}\right),\left(t_{0}, \ldots, t_{k-1}\right)\right)$ has $2 k$ points on each line and $2 q$ lines passing through each point. 


\section{Examples: Constructing a 6-Configuration, a 4-Configuration, and a $(4,6)$-Configuration}

The idea behind the general construction method is the following. Suppose we want to construct a $(2 q, 2 k)$-configuration. We begin with a single set of lines, labeled $L$, and construct $k$ symmetry classes of points so that each of the lines $L$ contains two points of each of the new classes, which are labeled $v_{0}, v_{1}, \ldots, v_{t_{k-1}}$. Next, we construct new symmetry classes of lines, with $q-2$ new pairs of lines-corresponding to each of the $q-2$ discrete parameters $s_{0}, s_{1}, \ldots, s_{q-2}$-emanating from each vertex. More precisely, we construct a set of lines of span $s_{j}$ with respect to each of the new vertex classes $v_{g}$. We label each new set of lines as $L_{g}^{p}$, for some $p$ in $S$ and some $g$ in $T$, where $p$ indicates that the line is of span $s_{p}$ with respect to the vertex set $v_{g}$.

Now, choose any two of the new sets of lines which are of the same span, say $L_{g}^{p}$ and $L_{h}^{p}$. As a consequence of the PCL, it turns out that two members of these two sets of lines are concurrent, so we can construct a new set of vertices $v_{g h}^{p}$, in which the upper index corresponds to the span of the lines which are concurrent, and the lower index corresponds to the vertices those lines emanated from. For example, a vertex labeled $v_{23}^{1}$ would lie on two lines labeled $L_{2}^{1}$ (which are themselves lines of span 1 with respect to the vertices labeled $v_{2}$ ) and two lines labeled $L_{3}^{1}$ (which are lines of span 1 with respect to the vertices $v_{3}$ ).

After constructing all these new sets of vertices, we again construct lines of each of the spans $s_{0}, s_{1}, \ldots, s_{q-2}$ with respect to each of the newly constructed vertex classes: for example, a line $L_{23}^{14}$ would be of span 4 with respect to the vertex class labeled $v_{23}^{1}$. A critical observation (a consequence of the PCL) for constructing these highly incident configurations is that this set of lines is also of span 1 with respect to the vertex class $v_{23}^{4}$.

In general, for each $n \leq \min \{q-1, k\}$ (each "step") we construct sets of points and sets of lines, and in the case where $q-1<k$, it is also necessary to construct extra sets of vertices. To do this, we consider the following. Given a pair of sets of lines $L_{\tau}^{\sigma}$ and $L_{\phi}^{\sigma}$, where $\tau, \phi$ and $\sigma$ are all of size $n$, if $\tau$ and $\phi$ differ by only a single element (i.e., their intersection is of size $n-1$ ), then two lines labeled $L_{\tau}^{\sigma}$ and two lines labeled $L_{\phi}^{\sigma}$ are concurrent (so it is possible to construct a new set of vertices). For example, two lines labeled $L_{456}^{123}$ and two lines labeled $L_{457}^{123}$ are concurrent, and we would label the new set of vertices as $v_{4567}^{123}$. As a consequence, if we consider the line $L_{456}^{123}$, then for any $z \in T$ other than $4,5,6$, we can construct a set of points labeled $v_{456 z}^{123}$, and each of those new points would lie on lines $L_{45 z}^{123}, L_{56 z}^{123}$ and $L_{46 z}^{123}$ as well.

Similarly, given a pair of sets of points $v_{\tau}^{\hat{\sigma}}$ and $v_{\tau}^{\hat{\rho}}$ where the size of $\hat{\rho}$ and $\hat{\sigma}$ is one less than the size of $\tau$, and $\hat{\rho}$ and $\hat{\sigma}$ differ by a single element, then two points labeled $v_{\tau}^{\hat{\sigma}}$ and two points labeled $v_{\tau}^{\hat{\rho}}$ are collinear. For example, two vertices labeled $v_{345}^{12}$ and two vertices labeled $v_{345}^{16}$ lie on a set of lines which may be labeled $L_{345}^{126}$. As with the points, for each $y \in S$, a point with label $v_{345}^{12}$ lies on a pair of lines with label $L_{345}^{12 y}$, and it also lies on pairs of lines with label $L_{34}^{12}, L_{35}^{12}, L_{45}^{12}$.

Of course, it is necessary to be careful to determine precisely which vertices are collinear and which lines are concurrent; this is rather technical and is done in Sect. 4. 


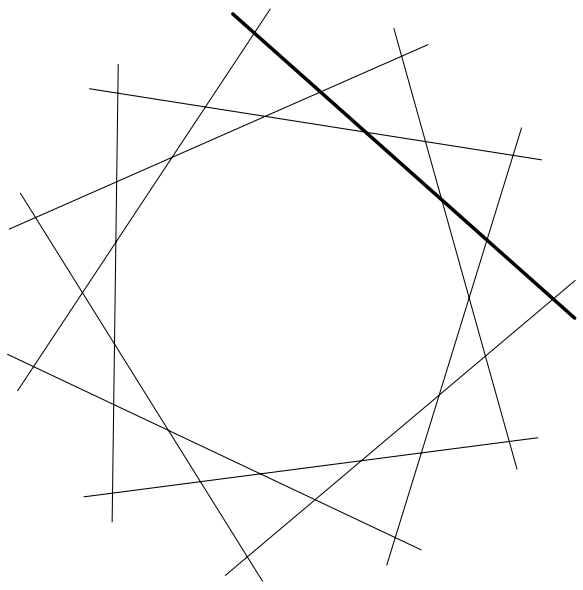

(A) The initial set of lines $L$.

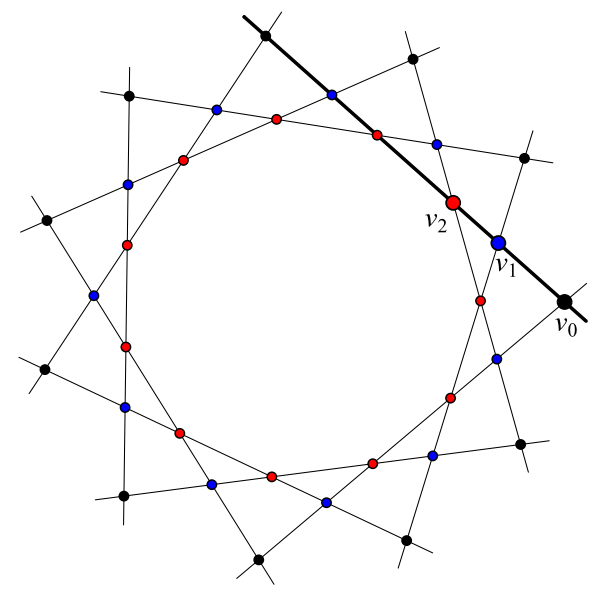

(B) Adding the first three sets of vertices.

Fig. 5 The initial set of lines and the vertices $v_{0}, v_{1}, v_{2}$ constructed in step 1 . The 0 th element of each set is shown larger

We walk through this construction method in its entirety by constructing a 6configuration $\left(110_{6}\right)$ with parameter list $((4,5),(3,2,1))$ and $m=11$; note that this is the smallest 6-configuration which may be constructed by this method, since we need $m / 2>2+3$. We illustrate the construction steps in Figs. 5-9. After that, as further illustration, we briefly apply the construction method to two smaller configurations, a well-known 4-configuration (Fig. 10) and a (4,6)-configuration (Fig. 11).

For notational convenience, for any set $\mathcal{S}$, let $p \mathcal{S}$ denote the set $\mathcal{S} \backslash\{p\}$, and let $\mathcal{S} g$ denote $\mathcal{S} \cup\{g\}$.

At each step $n$, we construct a set of points $v_{\tau}^{\hat{\sigma}}$ and a set of lines $L_{\tau}^{\sigma}$ for all subsets $\sigma \subset S$ and $\tau \subset T$ of size $n$ and all subsets $\hat{\sigma} \subset S$ of size $n-1$ :

- for each $g \in \tau$, define

$$
\left(v_{\tau}^{\hat{\sigma}}\right)_{i}:=\left(L_{g \tau}^{\hat{\sigma}}\right)_{i} \wedge\left(L_{g \tau}^{\hat{\sigma}}\right)_{i-t_{g}}
$$

- for each $p \in \sigma$, define

$$
\left(L_{\tau}^{\sigma}\right)_{i}:=\left(v_{\tau}^{p \sigma}\right)_{i} \vee\left(v_{\tau}^{p \sigma}\right)_{i+s_{p}}
$$

Step 0. Begin by constructing a set of lines $L$ which form the edges of a regular convex $m$-gon. See Fig. 5a.

Step 1. At this step, $n=1$. Consider points of the form $v_{\tau}^{\hat{\sigma}}$. Since $n=1$, the only choice for $\hat{\sigma}$ is the empty subset, and there are three subsets of $T$ of size 1 , namely $0,1,2$.

We must construct three sets of vertices (see Fig. 5b). 
- The vertices $v_{0}$ are the $t_{0}$ th points of intersection of the lines $L$ : since $t_{0}=3$, $\left(v_{0}\right)_{i}=(L)_{i} \wedge(L)_{i-t_{0}}=(L)_{i} \wedge(L)_{i-3}$.

- The vertices $v_{1}$ are the $t_{1}$ st points of intersection of the lines $L$ : since $t_{1}=2$, $\left(v_{1}\right)_{i}=(L)_{i} \wedge(L)_{i-t_{1}}=(L)_{i} \wedge(L)_{i-2}$.

- The vertices $v_{2}$ are the $t_{2}$ nd points of intersection of the lines $L$ : since $t_{2}=1$, $\left(v_{2}\right)_{i}=(L)_{i} \wedge(L)_{i-t_{2}}=(L)_{i} \wedge(L)_{i-1}$.

Now, beginning with each set of vertices we just constructed, we construct two sets of lines, corresponding to a choice of subset of size 1 from $S$. The two choices of subsets of $S$ are 0 and 1, so at this step we construct six new classes of lines. Precisely, for each vertex $v_{g}, g=0,1,2$, we construct a line of span $s_{0}$ and a line of span $s_{1}$ with respect to that set of vertices. These sets of lines are indexed downstairs corresponding to the vertex they emanate from and upstairs corresponding to their span. We construct the following lines (see Fig. 6).

- The lines $L_{0}^{0}$ are lines of $\operatorname{span} s_{0}=4$ with respect to $v_{0}$ : that is, $\left(L_{0}^{0}\right)_{i}=\left(v_{0}\right)_{i} \wedge$ $\left(v_{0}\right)_{i+4}$.

- The lines $L_{1}^{0}$ are lines of $\operatorname{span} s_{0}=4$ with respect to $v_{1}$ : that is, $\left(L_{1}^{0}\right)_{i}=\left(v_{1}\right)_{i} \wedge$ $\left(v_{1}\right)_{i+4}$.

- The lines $L_{2}^{0}$ are lines of $\operatorname{span} s_{0}=4$ with respect to $v_{2}$ : that is, $\left(L_{2}^{0}\right)_{i}=\left(v_{2}\right)_{i} \wedge$ $\left(v_{2}\right)_{i+4}$.

These lines are illustrated in Fig. 6b.

- The lines $L_{0}^{1}$ are lines of $\operatorname{span} s_{1}=5$ with respect to $v_{0}$ : that is, $\left(L_{0}^{0}\right)_{i}=\left(v_{0}\right)_{i} \wedge$ $\left(v_{0}\right)_{i+5}$.

- The lines $L_{1}^{1}$ are lines of $\operatorname{span} s_{1}=5$ with respect to $v_{1}$ : that is, $\left(L_{1}^{1}\right)_{i}=\left(v_{1}\right)_{i} \wedge$ $\left(v_{1}\right)_{i+5}$.

- The lines $L_{2}^{1}$ are lines of $\operatorname{span} s_{1}=5$ with respect to $v_{2}$ : that is, $\left(L_{2}^{1}\right)_{i}=\left(v_{2}\right)_{i} \wedge$ $\left(v_{2}\right)_{i+5}$.

These lines are illustrated in Fig. 6a.

Notice that at this point, line $L$ has 6 points on it (two each labeled $v_{0}, v_{1}, v_{2}$ ) and each point $v_{g}$ has 6 lines passing through it, two with label $L$, two with label $L_{g}^{0}$ and two with label $L_{g}^{1}$. However, each line $L_{g}^{p}$ has only two points on it, both with label $v_{g}$.

Step 2. In this step, we construct more vertices and lines, this time indexed by subsets $\hat{\sigma}$ of size $n-1=1$ and $\tau$ of size $n=2$ for vertices and $\sigma$ and $\tau$ of size $n=2$ for lines. We construct the vertices to be the intersections of lines which have the same upstairs index set and downstairs index sets which differ by a single element: the single differing element $r$ corresponds to choosing the $r$ th intersection point of the appropriate class of lines. That is, we construct six new sets of vertices, corresponding to a choice of $\hat{\sigma}$ of size 1 , so that $\hat{\sigma}$ is either 0 or 1 , and a choice of $\tau$ of size 2 , so that $\tau$ is 01,02 or 12 .

For example, consider the set of points with label $v_{01}^{0}$. In this case, $\tau=01$, so we define $v_{01}^{0}$ to be the $t_{1}$ st intersection of the lines $L_{0}^{0}$ (that is, $\left(v_{01}^{0}\right)_{i}=\left(L_{0}^{0}\right)_{i} \wedge$ $\left.\left(L_{0}^{0}\right)_{i-t_{1}}=\left(L_{0}^{0}\right)_{i} \wedge\left(L_{0}^{0}\right)_{i-2}\right)$ and also the $t_{0}$ th intersection of the lines $L_{1}^{0}$ (so that $\left.\left(v_{01}^{0}\right)_{i}=\left(L_{1}^{0}\right)_{i} \wedge\left(L_{1}^{0}\right)_{i-3}\right)$ : a main result of the proof of Theorem 5 is that these are the 
Fig. 6 The lines added in step 1. (a) Adding the lines $L_{0}^{0}$, $L_{1}^{0}, L_{2}^{0}$ of span 4. (b) Adding the lines $L_{0}^{1}, L_{1}^{1}, L_{2}^{1}$. The 0 th element of each set is shown larger

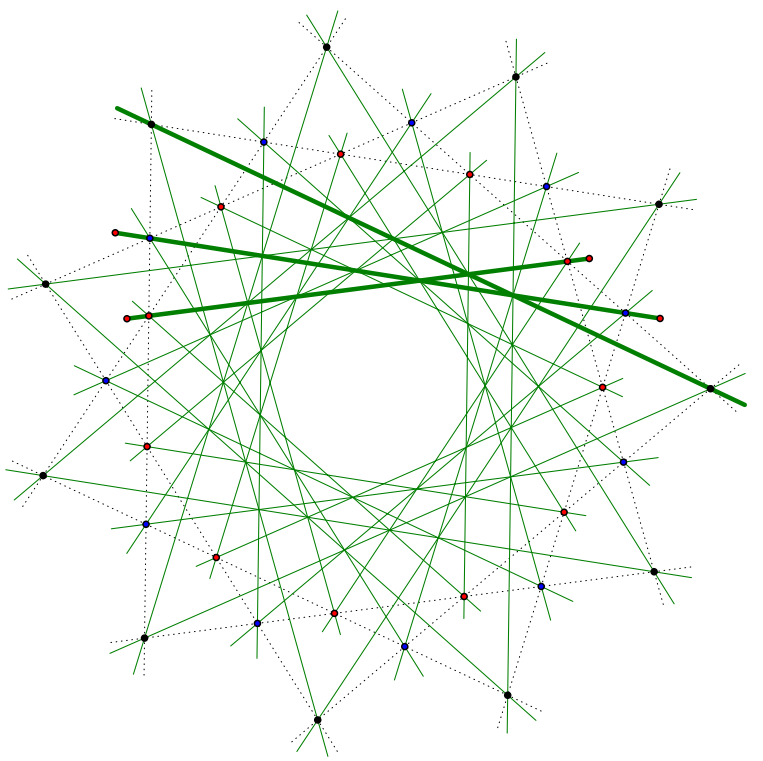

(A) Adding lines of span 4

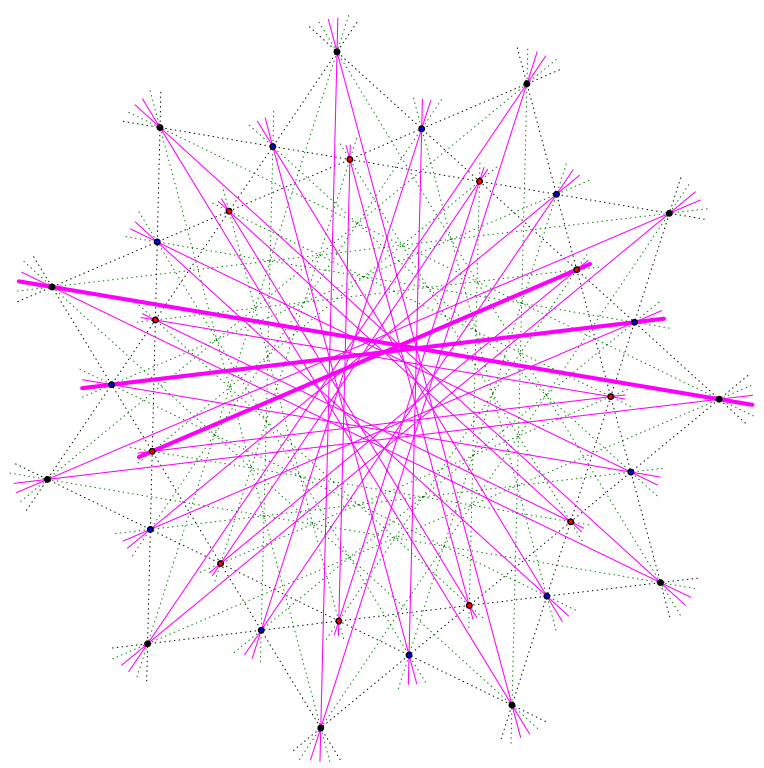

(в) Adding lines of span 5

same point, so that $\left(v_{01}^{0}\right)_{i}=\left(L_{0}^{0}\right)_{i} \wedge\left(L_{1}^{0}\right)_{i}$, which is more convenient for construction purposes.

Thus, we construct six new sets of vertices, illustrated in Fig. 7a: 
Fig. 7 Adding the three sets of lines and vertices from step 2 . (a) Adding the points $v_{01}^{0}, v_{02}^{0}$, $v_{12}^{0}$ (the outer large sets of points) and $v_{01}^{1}, v_{02}^{1}, v_{12}^{1}$ (the inner sets of points). (b) Adding the lines $L_{01}^{01}, L_{02}^{01}, L_{12}^{01}$. The 0th element of each set is shown larger

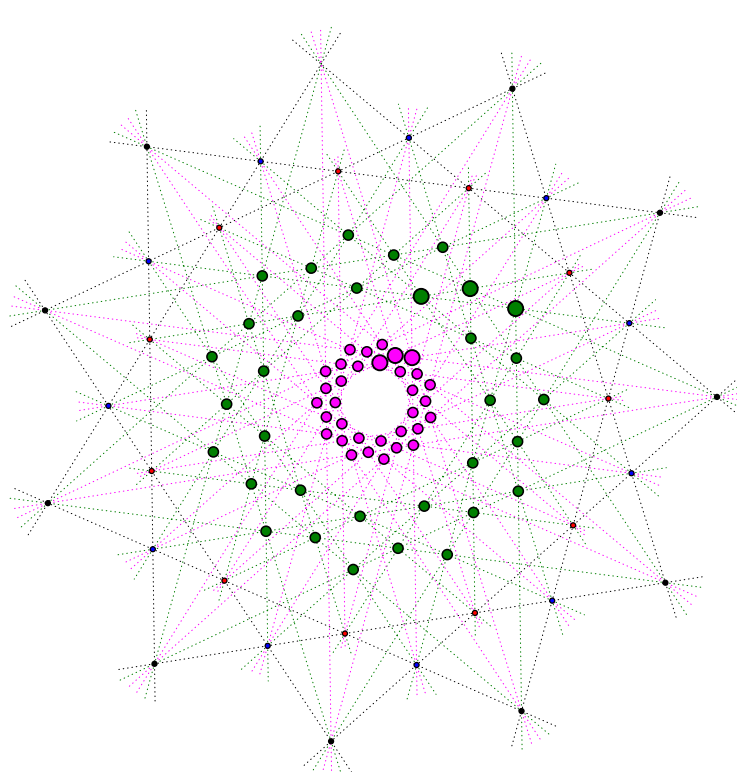

(A) Adding vertices in step 2

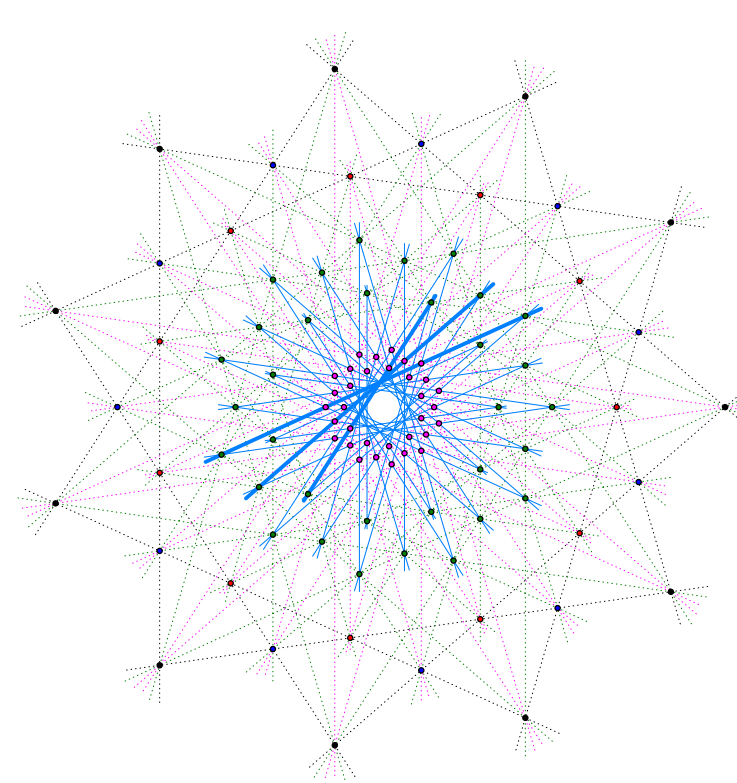

(B) Adding lines in step 2

- $v_{01}^{0}$ is the set of $t_{1}$ st intersection points of the lines $L_{0}^{0}$, so that $\left(v_{01}^{0}\right)_{i}=\left(L_{0}^{0}\right)_{i} \wedge$ $\left(L_{0}^{0}\right)_{i-t_{1}}$, or equivalently, $\left(v_{01}^{0}\right)_{i}=\left(L_{0}^{0}\right)_{i} \wedge\left(L_{1}^{0}\right)_{i}$; 
- $v_{02}^{0}$ is the set of $t_{2}$ nd intersection points of the lines $L_{0}^{0}$, so that $\left(v_{02}^{0}\right)_{i}=\left(L_{0}^{0}\right)_{i} \wedge$ $\left(L_{0}^{0}\right)_{i-t_{2}}$, or equivalently, $\left(v_{02}^{0}\right)_{i}=\left(L_{0}^{0}\right)_{i} \wedge\left(L_{2}^{0}\right)_{i}$;

- $v_{12}^{0}$ is the set of $t_{2}$ nd intersection points of the lines $L_{1}^{0}$, so that $\left(v_{12}^{0}\right)_{i}=\left(L_{1}^{0}\right)_{i} \wedge$ $\left(L_{1}^{0}\right)_{i-t_{2}}$, or equivalently, $\left(v_{12}^{0}\right)_{i}=\left(L_{1}^{0}\right)_{i} \wedge\left(L_{2}^{0}\right)_{i}$;

- $v_{01}^{1}$ is the set of $t_{1}$ st intersection points of the lines $L_{0}^{1}$, so that $\left(v_{01}^{1}\right)_{i}=\left(L_{0}^{1}\right)_{i} \wedge$ $\left(L_{0}^{1}\right)_{i-t_{1}}$, or equivalently, $\left(v_{01}^{1}\right)_{i}=\left(L_{0}^{1}\right)_{i} \wedge\left(L_{1}^{1}\right)_{i}$;

- $v_{02}^{1}$ is the set of $t_{2}$ nd intersection points of the lines $L_{0}^{1}$, so that $\left(v_{02}^{1}\right)_{i}=\left(L_{0}^{1}\right)_{i} \wedge$ $\left(L_{0}^{1}\right)_{i-t_{2}}$, or equivalently, $\left(v_{02}^{1}\right)_{i}=\left(L_{0}^{1}\right)_{i} \wedge\left(L_{2}^{1}\right)_{i}$;

- $v_{12}^{1}$ is the set of $t_{2}$ nd intersection points of the lines $L_{1}^{1}$, so that $\left(v_{12}^{1}\right)_{i}=\left(L_{1}^{1}\right)_{i} \wedge$ $\left(L_{1}^{1}\right)_{i-t_{2}}$, or equivalently, $\left(v_{12}^{1}\right)_{i}=\left(L_{1}^{1}\right)_{i} \wedge\left(L_{2}^{1}\right)_{i}$.

Notice that at this point, each line $L_{1}^{0}$, which already had two points labeled $v_{1}$ on it, now has two points labeled $v_{01}^{0}$ and two points labeled $v_{12}^{0}$ lying on it also. In fact, each line $L_{h}^{g}$ now has six points on it. On the other hand, each vertex with label $v_{01}^{0}$ lies on two lines with label $L_{0}^{0}$ and two lines with label $L_{1}^{0}$.

To construct new sets of lines, consider a set $\sigma$ of size $n=2$ and a set $\tau$ of size $n=2$. For example, consider $\sigma=01$ and $\tau=02$. We define $L_{02}^{01}$ to be the line of span $s_{1}$ with respect to the vertices $v_{02}^{0}$ and at the same time, we define $L_{02}^{01}$ to be the line of span $s_{0}$ with respect to the vertices $v_{02}^{1}$; the second main result of the proof of Theorem 5 is that these are the same lines, so that in fact, we could just as easily define $\left(L_{02}^{01}\right)_{i}$ to be the line connecting $\left(v_{02}^{0}\right)_{i}$ and $\left(v_{02}^{1}\right)_{i}$. We do this construction for each pair from $S$ (there's only one in this small case) and for each pair from $T$, namely $01,02,12$ to construct the line sets

- $L_{01}^{01}$ is the set of lines of $\operatorname{span} s_{1}=5$ with respect to $v_{01}^{0}$; alternately, $\left(L_{01}^{01}\right)_{i}=$ $\left(v_{01}^{0}\right)_{i} \vee\left(v_{01}^{1}\right)_{i}$

- $L_{02}^{01}$ is the set of lines of span $s_{1}=5$ with respect to $v_{02}^{0}$; alternately, $\left(L_{02}^{01}\right)_{i}=$ $\left(v_{02}^{0}\right)_{i} \vee\left(v_{02}^{1}\right)_{i}$

- $L_{12}^{01}$ is the set of lines of span $s_{1}=5$ with respect to $v_{12}^{0}$; alternately, $\left(L_{12}^{01}\right)_{i}=$ $\left(v_{12}^{0}\right)_{i} \vee\left(v_{12}^{1}\right)_{i}$

which are shown in Fig. $7 b$.

After these sets of lines are defined, notice that each point $v_{02}^{1}$ is incident with two lines with label $L_{0}^{1}$, two lines with label $L_{2}^{1}$, and two lines with label $L_{02}^{01}$ for a total of six lines, and in general, $v_{g h}^{p}$ lies on six lines.

Each line $L_{02}^{01}$, however, is only incident with two vertices of label $v_{02}^{0}$ and two vertices of label $v_{12}^{1}$ (and in general, each line $L_{g h}^{p r}$ is only incident with four points so far).

Final Step. Note that $\min \{q-1, k\}$ in this case is 2 , so we have finished all the steps in the iterative process. However, because we are in the special case when $q=k$, we need to construct an additional set of vertices, shown in Fig. 8:

- Define $v_{012}^{01}$ to be the $t_{2}$ nd intersection of lines $L_{01}^{01}$, so that $\left(v_{012}^{01}\right)_{i}=\left(L_{01}^{01}\right)_{i} \wedge$ $\left(L_{01}^{01}\right)_{i-t_{2}}=\left(L_{01}^{01}\right)_{i} \wedge\left(L_{01}^{01}\right)_{i-1}$; 
Fig. 8 Adding the final (innermost) set of vertices, shown larger

a consequence of the proof of Theorem 5 is that the three lines $\left(L_{01}^{01}\right)_{i},\left(L_{02}^{01}\right)_{i}$ and $\left(L_{12}^{01}\right)_{i}$ and the three lines $\left(L_{01}^{01}\right)_{i-t_{2}},\left(L_{02}^{01}\right)_{i-t_{1}}$ and $\left(L_{12}^{01}\right)_{i-t_{0}}$ all intersect at the point $\left(v_{012}^{01}\right)_{i}$. Moreover, each line $L_{g h}^{01}$ contains a pair of vertices with label $v_{012}^{01}$ in addition to the four vertices it is already incident with, for a total of six vertices on each of those lines as well.

Thus, we have constructed a 6-configuration with 10 symmetry classes of points and lines.

A second example - the simplest example possible - is in the case where $q=$ $k=2$, shown in Fig. 10. In this case, $m>6$, so we can choose $m=7$, and $\left(s_{0}, t_{0}, t_{1}\right)$ must therefore be a permutation of $\{1,2,3\}$. Choose $\left(s_{0}\right)=(3)$ and $\left(t_{0}, t_{1}\right)=(2,1)$. Then as before, construct lines $L$ (shown in black) that form the edges of a regular 7gon, and construct vertices $v_{0}$ (black) and $v_{1}$ (white) to be the second and first $\left(t_{0}=2\right.$ and $t_{1}=1$ ) intersection points of the lines $L$, respectively. Next, construct lines $L_{0}^{0}$ (gray) to be lines of $\operatorname{span} s_{0}=3$ with respect to the $v_{0}$ and lines $L_{1}^{0}$ (dotted) to be lines of span $s_{0}=3$ with respect to the $v_{1}$. Finally, construct vertices $v_{01}^{0}$ (gray) to be the second $\left(t_{0}=2\right)$ intersection point of the lines $L_{1}^{0}$ and also the first $\left(t_{1}=1\right)$ intersection point of the lines $L_{0}^{0}$. The resulting configuration is well-known: it is the 


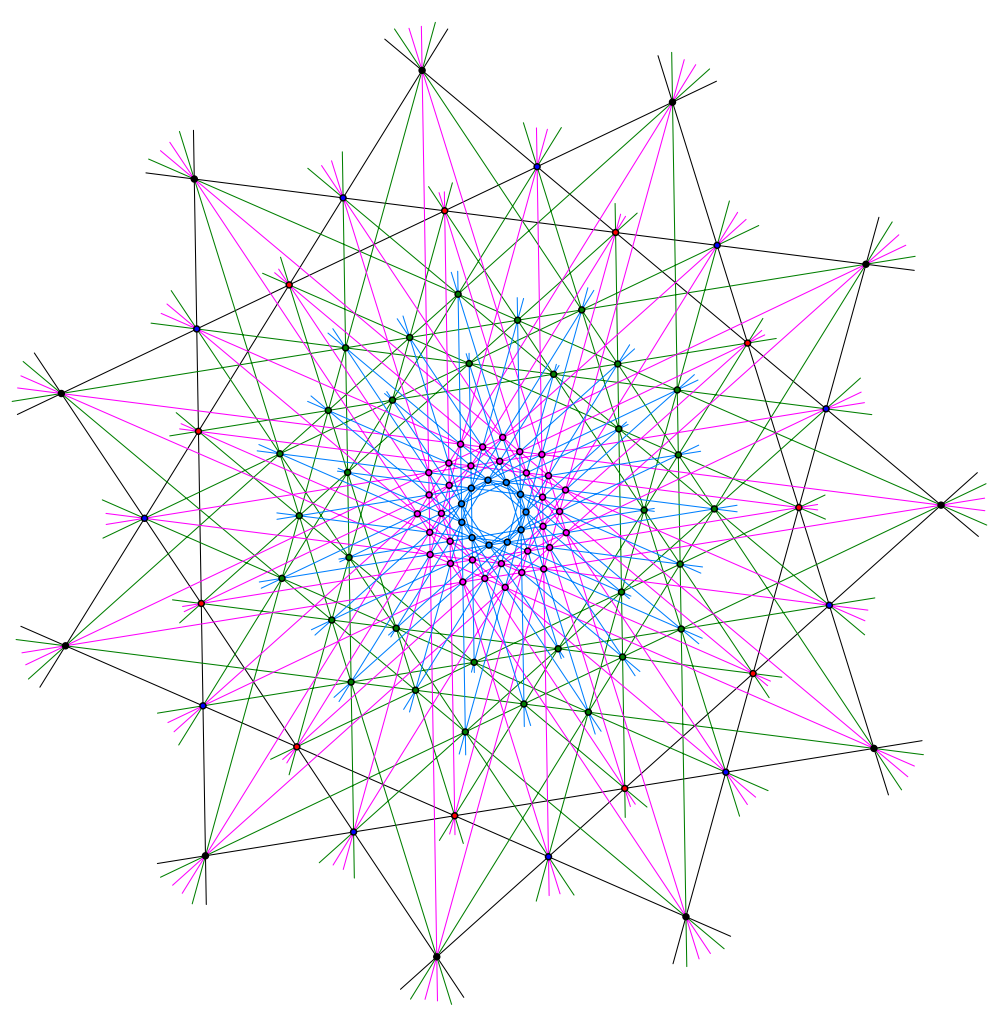

Fig. 9 The completed 6-configuration, $\left(110_{6}\right)$

$\left(21_{4}\right)$ configuration described in [15] which started much of the modern investigation into configurations!

In fact, in general the configuration constructed with symmetry group $d_{m}$ and parameter list $((a),(b, c))$ is well-understood: these are the trivial 3-astral 4configurations with symbol

$$
m \#(b, c ; a, b ; c, a) \text {. }
$$

See [14, Sect. 3.7] for more details on these configurations.

A final example of the construction technique-this time, constructing an unbalanced configuration-is shown in Fig. 11 , which shows a $(4,6)$-configuration with parameter list $((2),(3,4,1))$ and $m=9$. It is also shown (with different coloring) as Fig. 4.4.10(b) in [14]. In this example, there are four symmetry classes of lines and six symmetry classes of points. It is interesting to note that by adding diameters to this configuration, it is possible to construct a $(5,6)$-configuration, and by adding 9 points at infinity to the $(4,6)$-configuration, in the direction of each of the bands of parallel lines, it is possible to construct a $(4,7)$-configuration in the extended Euclidean plane. 
Fig. 10 A $\left(21_{4}\right)$ configuration, with $m=7$ and parameter list ((3), $(2,1))$, lines $L$ (black), $L_{0}^{0}$ (gray), $L_{1}^{0}$ (dotted), and points $v_{0}$ (black), $v_{1}$ (white), and $v_{01}^{0}$ (gray)

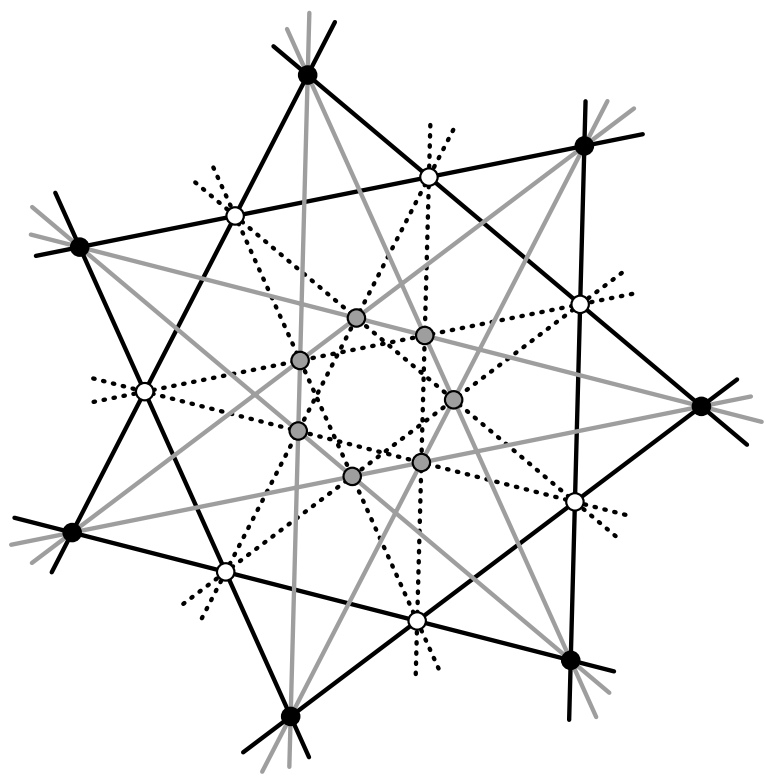

\section{Proof of Theorem 5}

In this section, we provide the details of the construction of a highly incident configuration with parameter list $\left(\left(s_{0}, s_{1}, \ldots, s_{q-2}\right),\left(t_{0}, t_{1}, \ldots, t_{k-1}\right)\right)$.

Proof of Theorem 5 Begin with the parameter list $\left(\left(s_{0}, \ldots, s_{q-2}\right),\left(t_{0}, \ldots, t_{k-1}\right)\right)$ and a choice of $m>2(q+k-1)$. Define $S=\{0,1, \ldots, q-2\}$ and $T=\{0,1, \ldots, k-1\}$ to be the index sets for the sets $\left\{s_{0}, s_{1}, \ldots, s_{q-2}\right\}$ and $\left\{t_{0}, t_{1}, \ldots, t_{k-1}\right\}$ respectively. We proceed iteratively. Let $N=\min \{q-1, k\}$.

For each $n$ with $0 \leq n \leq N$, for each subset $\hat{\sigma}$ and $\sigma$ of $S$ with $|\hat{\sigma}|=n-1,|\sigma|=n$ and for each subset $\tau \subseteq T$ with $|\tau|=n$, we construct a set of vertices $v_{\tau}^{\hat{\sigma}}$ and a set of lines $L_{\tau}^{\sigma}$.

\subsection{Beginning the Induction}

Initial setup: We define a set of lines $(L)_{i}$ to be the (extended) sides of a regular convex $m$-gon. We consider this set of lines $L$ to be indexed up and down by the empty subsets of $S$ and $T$ respectively, so there is $1=\left(\begin{array}{c}q-1 \\ 0\end{array}\right)\left(\begin{array}{l}k \\ 0\end{array}\right)$ set of lines constructed at this step.

Base Case: $n=1$ : Here, we choose $\hat{\sigma}$ of size $n-1=0$ (that is, the empty subset) and $\sigma, \tau$ of size $n=1$. We define vertices

$$
\left(v_{g}\right)_{i}=(L)_{i} \wedge(L)_{i-t_{g}}, \quad \text { for all } g \in T .
$$

Note that with this definition, $(L)_{i}=\left(v_{g}\right)_{i} \vee\left(v_{g}\right)_{i+t_{g}}$ (that is, the points $v_{g}$ are the $t_{g}$ th intersection points of the lines $L$, while the lines $L$ are the lines of span $t_{g}$ with respect to the vertices $v_{g}$ ). Because the parameters $t_{j}$ are all distinct, these vertex sets 


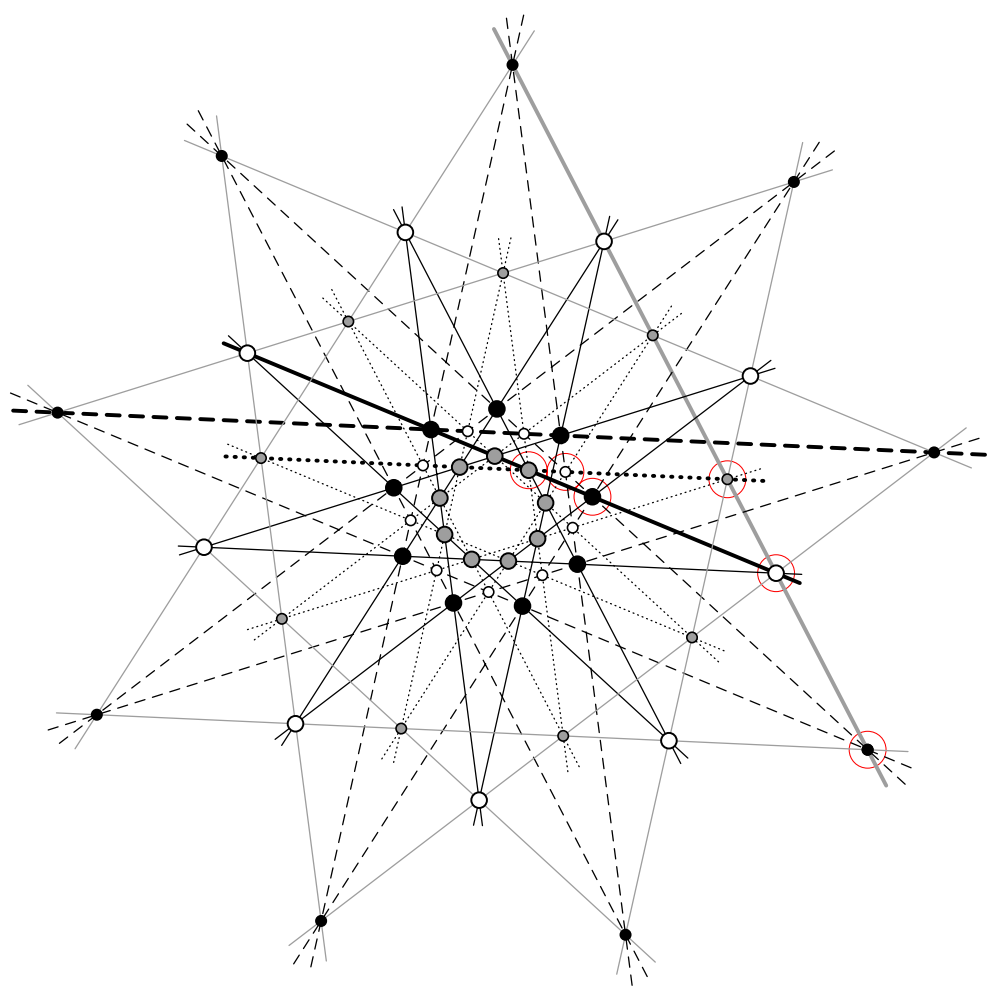

Fig. 11 A $(4,6)$-configuration $\left(54_{4}, 366\right)$ with parameter list $((2),(3,4,1))$ and $m=9$. In this diagram, the lines $L$ are solid black, the points $v_{0}$ are large and black, the points $v_{1}$ are large and white, and the points $v_{2}$ are large and gray. The lines $L_{0}^{0}$ are dashed, the lines $L_{1}^{0}$ are solid gray, and the lines $L_{2}^{0}$ are dotted. The vertices $v_{01}^{0}$ are smaller and black (on a gray-dashed intersection), the vertices $v_{02}^{0}$ are smaller and white (on a dotted-dashed intersection) and the vertices $v_{12}^{0}$ are smaller and gray (on a gray-dotted intersection). The 0th element of each set of lines is shown thicker, and the 0th element of each set of points is circled

are disjoint. There are $\left(\begin{array}{c}q-1 \\ 0\end{array}\right)\left(\begin{array}{l}k \\ 1\end{array}\right)$ sets of vertices constructed at this step, corresponding to the number of ways to index $v$ by choosing no elements from $S$ and one element from $T$.

(In practice, it is easier to begin the construction by constructing the vertices of a regular convex $m$-gon and labeling them cyclically as $\left(v_{0}\right)_{i}$, say by defining

$$
\left(v_{0}\right)_{i}=\left(\cos \left(\frac{2 \pi i}{m}\right), \sin \left(\frac{2 \pi i}{m}\right)\right)
$$

and then defining the lines $(L)_{i}=\left(v_{0}\right)_{i} \vee\left(v_{0}\right)_{i+t_{0}}$. Then using these lines $L$, construct the remainder of the vertices $v_{g}$ as defined above.)

Now we define for each $p \in S$ and $g \in T$ the new classes of lines

$$
\left(L_{g}^{p}\right)_{i}:=\left(v_{g}\right)_{i} \vee\left(v_{g}\right)_{i+s_{p}} .
$$


These lines $L_{g}^{p}$ are unambiguously defined: that is, for each choice of $p \in S$ and $g \in T$, a single set of lines is constructed. Because all the $s_{j}$ are distinct, the sets of lines are all different; because the parameters $s_{j}$ and $t_{p}$ are also distinct, the new lines constructed are distinct from the lines $L$.

Induction step: Let $\hat{\sigma} \subset S, \sigma \subset S$, and $\tau \subset T$ be sets with $|\hat{\sigma}|=n-1$ and $|\sigma|=$ $|\tau|=n$. Recall that for any set $\mathcal{S}, p \mathcal{S}$ denotes the set $\mathcal{S} \backslash\{p\}$ and $\mathcal{S} g$ denotes the set $\mathcal{S} \cup\{g\}$.

For each $g \in \tau$, define

$$
\left(v_{\tau}^{\hat{\sigma}}\right)_{i}:=\left(L_{g \tau}^{\hat{\sigma}}\right)_{i} \wedge\left(L_{g \tau}^{\hat{\sigma}}\right)_{i-t_{g}},
$$

and for each $p \in \sigma$, define

$$
\left(L_{\tau}^{\sigma}\right)_{i}:=\left(v_{\tau}^{p \sigma}\right)_{i} \vee\left(v_{\tau}^{p \sigma}\right)_{i+s_{p}} .
$$

We use induction and the PCL and LCL to show that these definitions are independent of the choice of $p \in \sigma$ and the choice of $g \in \tau$, so that a single set of lines or vertices is constructed for each choice of $\hat{\sigma}, \tau$ or $\sigma, \tau$, respectively.

\subsection{The Inductive Step}

As the induction hypothesis, we assume that for any sets $\sigma, \tau$ with cardinality $1<$ $a<n$ and any set $\hat{\sigma}$ with cardinality $a-1$, that there is a single set of lines or vertices constructed as above.

Induction Case 1: vertices. In this case, $|\hat{\sigma}| \geq 1$ and $|\tau| \geq 2$. To show that $\left(v_{\tau}^{\hat{\sigma}}\right)_{i}$ is well-defined, we must show that for any $g, h \in \tau$,

$$
\left(L_{g \tau}^{\hat{\sigma}}\right)_{i} \wedge\left(L_{g \tau}^{\hat{\sigma}}\right)_{i-t_{h}}=\left(L_{h \tau}^{\hat{\sigma}}\right)_{i} \wedge\left(L_{h \tau}^{\hat{\sigma}}\right)_{i-t_{g}}
$$

that is, we must show that the four lines

$$
\left(L_{g \tau}^{\hat{\sigma}}\right)_{i},\left(L_{g \tau}^{\hat{\sigma}}\right)_{i-t_{h}},\left(L_{h \tau}^{\hat{\sigma}}\right)_{i},\left(L_{h \tau}^{\hat{\sigma}}\right)_{i-t_{g}}
$$

intersect in a single point.

We apply the PCL, with $\alpha=t_{g}, \beta=s_{p}, \delta=t_{h}$, and definitions

$$
\begin{aligned}
& u_{i}=\left(v_{h \tau}^{p \hat{\sigma}}\right)_{i}, \\
& \Theta_{i}=u_{i} \vee u_{i+\alpha}=\left(v_{h \tau}^{p \hat{\sigma}}\right)_{i} \vee\left(v_{h \tau}^{p \hat{\sigma}}\right)_{i+t_{g}}=\left(L_{g h \tau}^{p \hat{\sigma}}\right)_{i}, \\
& \Psi_{i}=u_{i} \vee u_{i+\beta}=\left(v_{h \tau}^{p \hat{\sigma}}\right)_{i} \vee\left(v_{h \tau}^{p \hat{\sigma}}\right)_{i+s_{p}}=\left(L_{h \tau}^{\hat{\sigma}}\right)_{i} \quad \text { (by the induction hypothesis), } \\
& x_{i}=\Theta_{i} \wedge \Theta_{i-\delta}=\left(L_{g h \tau}^{p \hat{\sigma}}\right)_{i} \wedge\left(L_{g h \tau}^{p \hat{\sigma}}\right)_{i+t_{h}}=\left(v_{g \tau}^{p \hat{\sigma}}\right)_{i} \quad \text { (by the induction hypothesis), } \\
& \Gamma_{i}=x_{i} \vee x_{i+\beta}=\left(v_{g \tau}^{p \hat{\sigma}}\right)_{i} \vee\left(v_{g \tau}^{p \hat{\sigma}}\right)_{i+s_{p}}=\left(L_{g \tau}^{\hat{\sigma}}\right)_{i} \quad \text { (by the induction hypothesis) }
\end{aligned}
$$

and conclude that each point

$$
y_{i}:=\left(L_{g \tau}^{\hat{\sigma}}\right)_{i} \wedge\left(L_{h \tau}^{\hat{\sigma}}\right)_{i}
$$


also lies on the lines $\left(L_{g \tau}^{\hat{\sigma}}\right)_{i-t_{h}}$ and $\left(L_{h \tau}^{\hat{\sigma}}\right)_{i-t_{g}}$. That is, the four lines

$$
\left(L_{g \tau}^{\hat{\sigma}}\right)_{i},\left(L_{g \tau}^{\hat{\sigma}}\right)_{i-t_{h}},\left(L_{h \tau}^{\hat{\sigma}}\right)_{i},\left(L_{h \tau}^{\hat{\sigma}}\right)_{i-t_{g}}
$$

are concurrent at the point $\left(v_{\tau}^{\hat{\sigma}}\right)_{i}$.

Induction Case 2: lines. In this case, we assume $|\sigma| \geq 2$ and $|\tau| \geq 2$. To show that $\left(L_{\tau}^{\sigma}\right)_{i}$ is well-defined, it suffices to show that for any distinct $p, r \in \sigma$,

$$
\left(L_{\tau}^{\sigma}\right)_{i}=\left(v_{\tau}^{p \sigma}\right)_{i} \vee\left(v_{\tau}^{p \sigma}\right)_{i+s_{p}}=\left(v_{\tau}^{r \sigma}\right)_{i} \vee\left(v_{\tau}^{r \sigma}\right)_{i+s_{r}}
$$

that is, we must show that $\left(v_{\tau}^{p \sigma}\right)_{i},\left(v_{\tau}^{p \sigma}\right)_{i+s_{p}},\left(v_{\tau}^{r \sigma}\right)_{i},\left(v_{\tau}^{r \sigma}\right)_{i+s_{r}}$ are collinear.

We choose an arbitrary $g \in \tau$ and apply the LCL with $\alpha=-s_{r}, \beta=-t_{g}, \delta=s_{p}$, as follows. Define

$$
\begin{aligned}
& U_{i}=\left(L_{g \tau}^{p \sigma}\right)_{i}, \\
& \theta_{i}=U_{i} \wedge U_{i+\alpha}=\left(L_{g \tau}^{p \sigma}\right)_{i} \wedge\left(L_{g \tau}^{p \sigma}\right)_{i-s_{r}}=\left(v_{g \tau}^{p r \sigma}\right)_{i}, \\
& X_{i}=\theta_{i} \vee \theta_{i+\delta}=\left(v_{g \tau}^{p r \sigma}\right)_{i} \vee\left(v_{g \tau}^{p r \sigma}\right)_{i+s_{p}}=\left(L_{g \tau}^{r \sigma}\right)_{i} \quad \text { (by the induction hypothesis), } \\
& \psi_{i}=U_{i} \wedge U_{i+\beta}=\left(L_{g \tau}^{p \sigma}\right)_{i} \wedge\left(L_{g \tau}^{p \sigma}\right)_{i-t_{g}}=\left(v_{\tau}^{p \sigma}\right)_{i} \quad \text { (by the induction hypothesis), } \\
& \gamma_{i}=X_{i} \wedge X_{i+\beta}=\left(L_{g \tau}^{r \sigma}\right)_{i} \wedge\left(L_{g \tau}^{r \sigma}\right)_{i-t_{g}}=\left(v_{\tau}^{r \sigma}\right)_{i} \quad \text { (by the induction hypothesis) } .
\end{aligned}
$$

To show that

$$
\left(L_{g \tau}^{p \sigma}\right)_{i} \wedge\left(L_{g \tau}^{p \sigma}\right)_{i+s_{r}}=\left(v_{g \tau}^{p r \sigma}\right)_{i},
$$

note that since $|\operatorname{pr} \sigma|=n-2,|p \sigma|=n-1$, and $|g \tau|=n-1$, by induction,

$$
\left(L_{g \tau}^{p \sigma}\right)_{i}=\left(v_{g \tau}^{r p \sigma}\right)_{i} \vee\left(v_{g \tau}^{r p \sigma}\right)_{i+s_{r}}
$$

or alternately, from the point of view of the line intersections, that

$$
\left(v_{g \tau}^{r p \sigma}\right)_{i}=\left(L_{g \tau}^{p \sigma}\right)_{i} \wedge\left(L_{g \tau}^{p \sigma}\right)_{i-s_{r}} .
$$

We conclude via the LCL that the points

$$
\left\{\gamma_{i}, \gamma_{i-\alpha}, \psi_{i}, \psi_{i+\delta}\right\}=\left\{\left(v_{\tau}^{r \sigma}\right)_{i},\left(v_{\tau}^{r \sigma}\right)_{i+s_{r}},\left(v_{\tau}^{p \sigma}\right)_{i},\left(v_{\tau}^{p \sigma}\right)_{i+s_{p}}\right\}
$$

are collinear.

\subsection{Analysis of the Points and Lines}

It remains to count how many points and lines have been constructed at each step $n$, and show that the points and lines constructed at each step have the required incidences. Suppose $n<N$.

By construction, line $(L)_{i}$ contains $k$ vertices, $\left(v_{0}\right)_{i},\left(v_{1}\right)_{i}, \ldots,\left(v_{k-1}\right)_{i}$, and there were $\left(\begin{array}{c}q-1 \\ 0\end{array}\right)\left(\begin{array}{l}k \\ 0\end{array}\right)$ ways to choose this set of lines. 
For $n \geq 1$, at step $n$, for each choice of $\sigma, \tau$ of size $n$ and each choice of $\hat{\sigma}$ of size $n-1$, we constructed lines $\left(L_{\tau}^{\sigma}\right)_{i}$ and points $\left(v_{\tau}^{\hat{\sigma}}\right)_{i}$. Thus, $\left(\begin{array}{c}q-1 \\ n\end{array}\right)\left(\begin{array}{l}k \\ n\end{array}\right)$ new sets of lines and $\left(\begin{array}{l}q-1 \\ n-1\end{array}\right)\left(\begin{array}{l}k \\ n\end{array}\right)$ new sets of vertices are constructed at each step.

Now let $\hat{\sigma}$ be any subset of $S$ of size $n-1$ and consider a vertex $\left(v_{\tau}^{\hat{\sigma}}\right)_{i}:=\left(L_{g \tau}^{\hat{\sigma}}\right)_{i} \wedge$ $\left(L_{g \tau}^{\hat{\sigma}}\right)_{i-t_{g}}$. Each vertex $\left(v_{\tau}^{\hat{\sigma}}\right)_{i}$ lies on the pair of lines $\left\{\left(L_{g \tau}^{p \sigma}\right)_{i},\left(L_{g \tau}^{p \sigma}\right)_{i-t_{g}}\right\}$ for each $g \in \tau$, for a total of $n$ such lines. In addition, the vertex $\left(v_{\tau}^{\hat{\sigma}}\right)_{i}$ lies on all the pairs of lines $\left\{\left(L_{\tau}^{\hat{\sigma} p}\right)_{i},\left(L_{\tau}^{\hat{\sigma} p}\right)_{i-t_{p}}\right\}$ for each $p \in S$ with $p \notin \hat{\sigma}$; there are $(q-1)-(n-1)$ of these pairs, for a total of

$$
2(n+(q-1)-(n-1))=2 q
$$

lines intersecting at $\left(v_{\tau}^{\hat{\sigma}}\right)_{i}$.

Finally, consider a line $\left(L_{\tau}^{\sigma}\right)_{i}:=\left(v_{\tau}^{p \sigma}\right)_{i} \vee\left(v_{\tau}^{p \sigma}\right)_{i+s_{p}}$. For each $h \notin \tau$,

$$
\left(v_{\tau h}^{\sigma}\right)=\left(L_{\tau}^{\sigma}\right)_{i} \wedge\left(L_{\tau}^{\sigma}\right)_{i+t_{h}}
$$

In other words, each line $\left(L_{\tau}^{\sigma}\right)_{i}$ contains the pair of vertices $\left\{\left(v_{\tau}^{p \sigma}\right),\left(v_{\tau}^{p \sigma}\right)_{i+s_{p}}\right\}$ for each $p \in \sigma$ and the pair of vertices $\left\{\left(v_{\tau h}^{\sigma}\right),\left(v_{\tau h}^{\sigma}\right)_{i+t_{h}}\right\}$ for each $h \notin \tau$. Since $|\sigma|=$ $|\tau|=n$, there are a total of

$$
2(n+(k-n))=2 k
$$

vertices incident with each line $\left(L_{\tau}^{\sigma}\right)_{i}$.

\subsection{Finishing the Iterative Process}

We need to determine the situation when $n=N=\min \{q-1, k\}$. We have two cases.

Case 1: $N=q-1<k$. Let $\sigma=S$ and let $\tau$ be a proper subset of $T$ of size $N$ (since $k>N$ ).

In this case, at step $N$, we construct a set of vertices $\left(v_{\tau}^{p S}\right)_{i}$ and a set of lines $\left(L_{\tau}^{S}\right)_{i}$. Each vertex $\left(v_{\tau}^{p S}\right)_{i}$ is incident with the line pairs $\left\{\left(L_{g \tau}^{p S}\right)_{i},\left(L_{g \tau}^{p S}\right)_{i-t_{g}}\right\}$ for each $g \in \tau$ and the single line pair $\left\{\left(L_{\tau}^{S}\right)_{i},\left(L_{\tau}^{S}\right)_{i-s_{p}}\right\}$ for a total of $2 q$ lines incident with the vertex.

However, while each line $\left(L_{\tau}^{S}\right)_{i}$ is incident with the pair of vertices $\left\{\left(v_{\tau}^{p S}\right)_{i}\right.$, $\left.\left(v_{\tau}^{p S}\right)_{i+s_{p}}\right\}$ for each $p \in S$, there are only $q-1$ such pairs. Since $|\tau|<k$, we can construct additional sets of vertices

$$
\left(v_{\tau h}^{S}\right)_{i}=\left(L_{\tau}^{S}\right)_{i} \wedge\left(L_{\tau}^{S}\right)_{i-t_{h}}
$$

for each $h \notin \tau$ so that line $\left(L_{\tau}^{S}\right)_{i}$ is also incident with the $k-N$ pairs of vertices $\left\{\left(v_{\tau h}^{S}\right)_{i},\left(v_{\tau h}^{S}\right)_{i+t_{h}}\right\}$ for a total of $2((q-1)+(k-(q-1)))=2 k$ vertices lying on each line.

Finally, for each set $\bar{\tau} \subset T$ of size $N+1$ the new vertices $\left(v_{\bar{\tau}}^{S}\right)_{i}$ lie on the line pairs $\left\{\left(L_{\tau}^{S}\right)_{i},\left(L_{\tau}^{S}\right)_{i-t_{h}}\right\}$ for each $h \in \bar{\tau}$; there are $(q-1)+1$ line pairs, for a total of $2 q$ lines incident with each new vertex. 
In this case, we constructed $\left(\begin{array}{c}q-1 \\ q-1\end{array}\right)\left(\begin{array}{c}k \\ q-1\end{array}\right)$ new sets of lines and $\left(\begin{array}{c}q-1 \\ q-2\end{array}\right)\left(\begin{array}{c}k \\ q-1\end{array}\right)+$ $\left(\begin{array}{l}q-1 \\ q-1\end{array}\right)\left(\begin{array}{l}k \\ q\end{array}\right)$ sets of vertices.

Note that if $q-1=k-1$, then the configuration constructed is a $2 k$-configuration.

Case 2: $N=k \leq q-1$. Let $\sigma$ be a subset of $S$ of size $N$ and let $\tau=T$. At step $N$, we construct vertices $\left(v_{T}^{p \sigma}\right)_{i}$ and lines $\left(L_{T}^{\sigma}\right)_{i}$. Let $\hat{\sigma} \subset S$ of size $N-1$.

Each vertex $\left(v_{T}^{p \sigma}\right)_{i}$ lies on the pairs of lines $\left\{\left(L_{g T}^{p \sigma}\right)_{i},\left(L_{g T}^{p \sigma}\right)_{i-t_{g}}\right\}$ for each $g \in T-$ there are $N=k$ of these-and on the pairs $\left\{\left(L_{T}^{\hat{\sigma} p}\right)_{i},\left(L_{T}^{\hat{\sigma} p}\right)_{i-s_{p}}\right\}$ for each $p \notin \hat{\sigma}$; since $|\hat{\sigma}|=N-1$, there are $(q-1)-(N-1)$ pairs of this second type, for a total of

$$
2(N+(q-1)-(N-1))=2 q
$$

lines passing through each vertex $\left(v_{T}^{p \sigma}\right)_{i}$.

Each line of the form $\left(L_{T}^{\sigma}\right)_{i}$ is incident with the vertex pairs $\left\{\left(v_{T}^{p \sigma}\right)_{i},\left(v_{T}^{p \sigma}\right)_{i+s_{p}}\right\}$ for each $p \in \sigma$; since $|\sigma|=N=k$, each line is incident with $2 k$ vertices.

In this case, at step $N$ we constructed $\left(\begin{array}{c}q-1 \\ k\end{array}\right)\left(\begin{array}{l}k \\ k\end{array}\right)$ sets of lines and $\left(\begin{array}{c}q-1 \\ k-1\end{array}\right)\left(\begin{array}{c}q-1 \\ k\end{array}\right)$ sets of vertices.

\subsection{Counting Symmetry Classes}

Again, there are two cases, corresponding to the value of $N$. To sum up the total number of classes of lines and points, we use Lemmas 6 and 7, stated and proved below.

Case 1: $N=q-1<k$. The total number of symmetry classes of lines is

$$
\sum_{n=0}^{q-1}\left(\begin{array}{c}
q-1 \\
n
\end{array}\right)\left(\begin{array}{l}
k \\
n
\end{array}\right)=\left(\begin{array}{c}
q+k-1 \\
q-1
\end{array}\right),
$$

using Lemma 6 with $a=k, b=q-1, c=0$, and the total number of symmetry classes of points is

$$
\sum_{n=0}^{q-1}\left(\begin{array}{c}
q-1 \\
n
\end{array}\right)\left(\begin{array}{c}
k \\
n+1
\end{array}\right)=\left(\begin{array}{c}
q+k-1 \\
q
\end{array}\right),
$$

using Lemma 7 with $a=q-1, b=k, c=1$.

Case 2: $N=k \leq q-1$. The total number of symmetry classes of lines is

$$
\sum_{n=0}^{k}\left(\begin{array}{c}
q-1 \\
n
\end{array}\right)\left(\begin{array}{l}
k \\
n
\end{array}\right)=\left(\begin{array}{c}
q-1+k \\
k
\end{array}\right)
$$

and the total number of symmetry classes of points is

$$
\sum_{n=0}^{k-1}\left(\begin{array}{c}
q-1 \\
n
\end{array}\right)\left(\begin{array}{c}
k \\
n+1
\end{array}\right)=\left(\begin{array}{c}
q-1+k \\
k-1
\end{array}\right)
$$




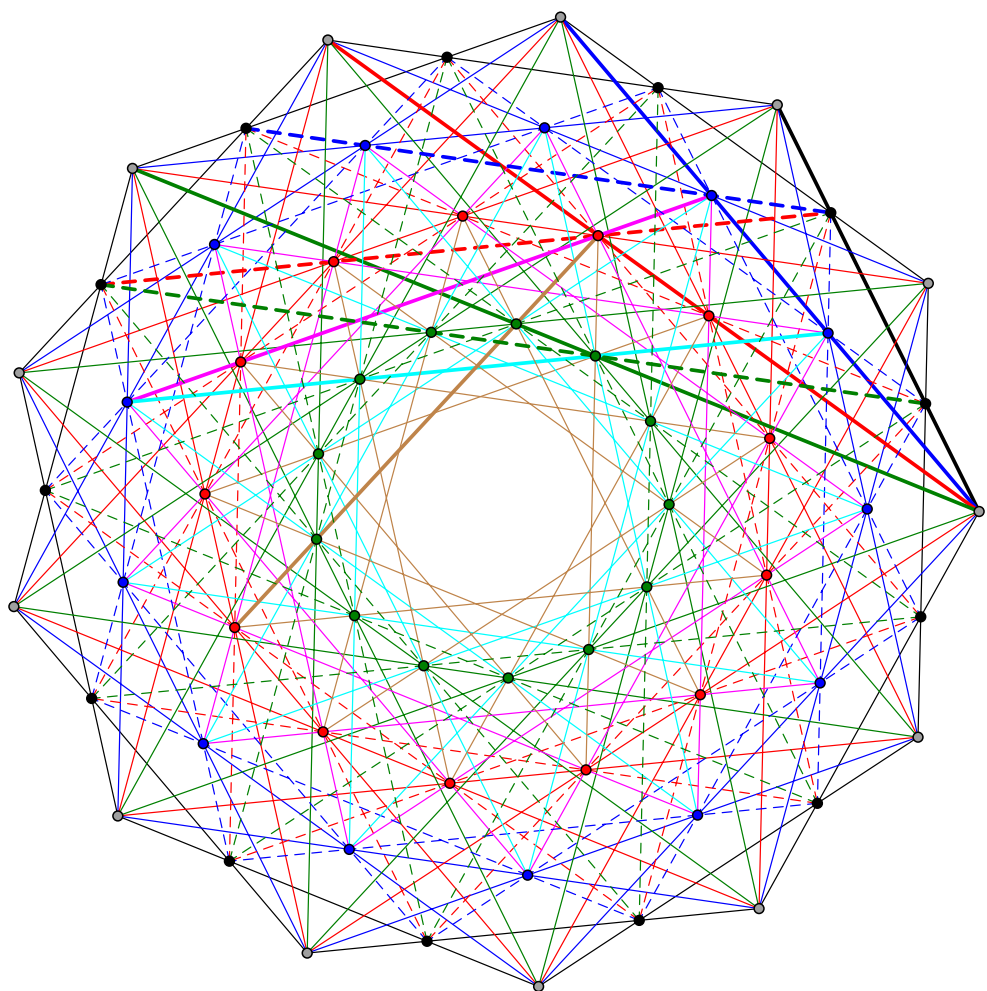

Fig. 12 A $(8,4)$-configuration $\left(65_{8}, 130_{4}\right)$ with parameter list $((3,4,5),(2,1))$ and $m=13$

again using Lemma 6 with $a=q-1, b=k$ and $c=0$ (lines) or $c=1$ (points). Finally, by the construction method, the resulting configuration has the dihedral symmetry of an $m$-gon.

Lemma 6 If $0 \leq c \leq b$ and $b-c \leq a$, then

$$
\sum_{n=0}^{b-c}\left(\begin{array}{l}
a \\
n
\end{array}\right)\left(\begin{array}{c}
b \\
n+c
\end{array}\right)=\left(\begin{array}{l}
a+b \\
b-c
\end{array}\right)
$$

Proof Consider a set of $a+b$ elements, of which $a$ are colored red and $b$ are colored blue. To choose $b-c$ elements, we either choose them directly from the set in $\left(\begin{array}{l}a+b \\ b-c\end{array}\right)$ ways, or for each $n \leq b-c$ we choose $n$ red items to include and $n+c$ blue items to exclude, choosing a total of $n+(b-(n+c))=b-c$ items at each step.

Lemma 7 If $0 \leq c \leq b$ and $a \leq b-c$, then

$$
\sum_{n=0}^{a}\left(\begin{array}{l}
a \\
n
\end{array}\right)\left(\begin{array}{c}
b \\
n+c
\end{array}\right)=\left(\begin{array}{l}
a+b \\
a+c
\end{array}\right)
$$




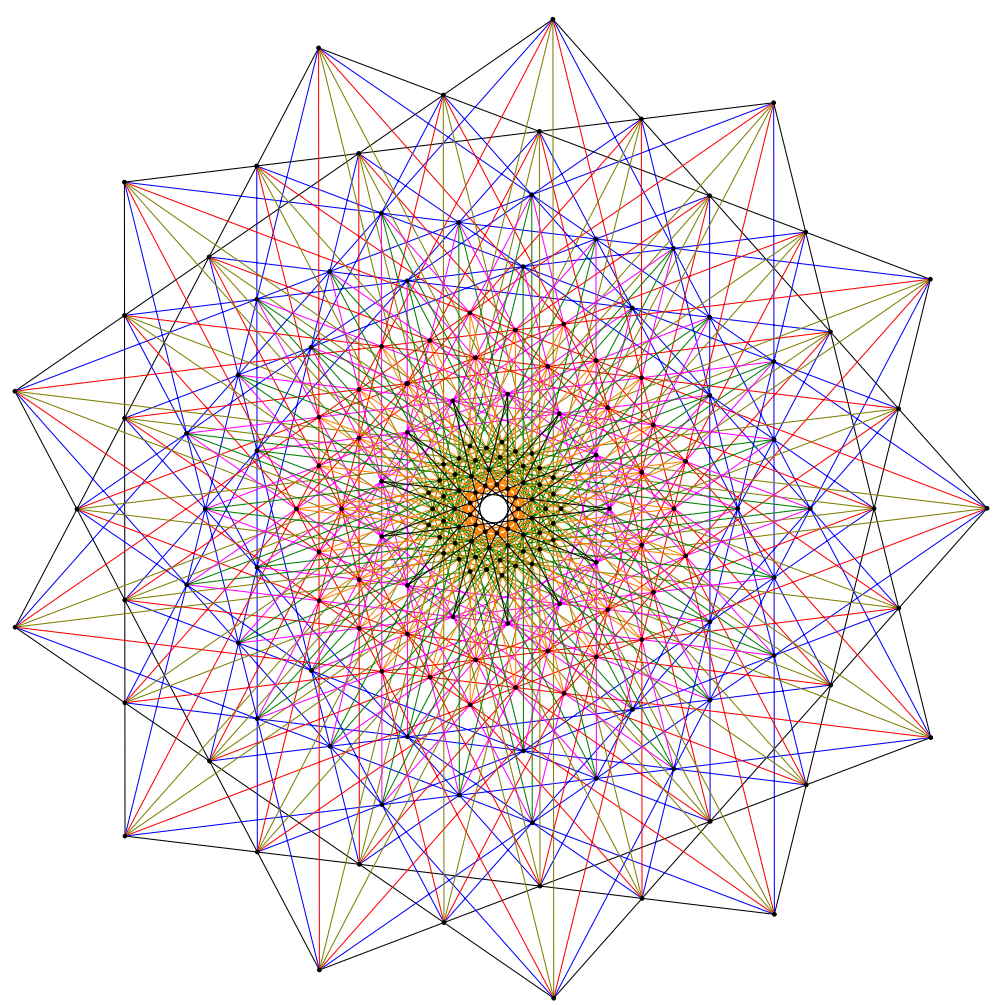

Fig. 13 An $(8,6)$-configuration $\left(195_{8}, 260_{6}\right)$

Proof Again consider a set of $a+b$ elements, of which $a$ are colored red and $b$ are colored blue. To choose $a+c$ elements, we either choose them directly from the set in $\left(\begin{array}{l}a+b \\ a+c\end{array}\right)$ ways, or for each $n \leq a$ we choose $n$ red items to exclude and $n+c$ blue items to include, choosing a total of $(a-n)+(n+c)=a+c$ items at each step.

One nice consequence of Theorem 5 is that we can now answer definitively the question of the existence of any $2 k$-configurations with non-trivial symmetry for $k>2$, which prior to this theorem was essentially unknown, except for a few highly complicated 6- and 8-configurations.

Corollary 8 For every $k \geq 2$, it is possible to construct geometric $2 k$-configurations which have $\left(\begin{array}{c}2 k-1 \\ k\end{array}\right)$ symmetry classes of points and lines.

Proof We apply Theorem 5 with $q=k$. In this case, we construct $\left(\begin{array}{c}2 k-1 \\ k\end{array}\right)$ symmetry classes of points and $\left(\begin{array}{c}2 k-1 \\ k-1\end{array}\right)$ symmetry classes of lines. Since $\left(\begin{array}{c}2 k-1 \\ k\end{array}\right)=\left(\begin{array}{c}2 k-1 \\ (2 k-1)-k\end{array}\right)=$ $\left(\begin{array}{c}2 k-1 \\ k-1\end{array}\right)$, the symmetry classes are of the same size. 


\section{Picture Gallery}

The 6-configuration with parameter list $((3,2),(5,1,4))$ and $m=11$ shown in Fig. 9 is also shown as Fig. 4.2.1 in [14], although with different coloring; it has $\left(\begin{array}{l}5 \\ 3\end{array}\right)=10$ symmetry classes of points and lines.

Figure 12 shows an $(8,4)$-configuration $\left(65_{8}, 130_{4}\right)$ with parameter list $((3,4,5)$, $(2,1))$ and $m=13$.

Finally, Fig. 13 shows an $(8,6)$-configuration $\left(195_{8}, 260_{6}\right)$, with $q-1=3$ and $k=3$, with $\left(\begin{array}{l}6 \\ 2\end{array}\right)=15$ classes of points and $\left(\begin{array}{l}6 \\ 3\end{array}\right)=20$ classes of lines. In this case, $m=13$ and the parameter list is $((4,5,6),(3,2,1))$.

\section{References}

1. Berman, L.W.: A characterization of astral $\left(n_{4}\right)$ configurations. Discrete Comput. Geom. 26(4), 603612 (2001)

2. Berman, L.W.: Even astral configurations. Electron. J. Comb. 11(1) (2004). Research Paper 37, 23 pp. (electronic)

3. Berman, L.W.: Movable $\left(n_{4}\right)$ configurations. Electron. J. Comb. 13(1) (2006). Research Paper 104, $30 \mathrm{pp}$. (electronic)

4. Berman, L.W.: Some results on odd astral configurations. Electron. J. Comb. 13(1) (2006). Research Paper 27, 31 pp. (electronic)

5. Berman, L.W., Bokowski, J.: Linear astral $\left(n_{5}\right)$ configurations with dihedral symmetry. Eur. J. Comb. 29(8), 1831-1842 (2008)

6. Berman, L.W., Ng, L.: Constructing 5-configurations with chiral symmetry. Electron. J. Comb. 17(1) (2010). Research Paper 2, 14

7. Berman, L.W., Bokowski, J., Grünbaum, B., Pisanski, T.: Geometric "floral" configurations. Can. Math. Bull. 52(3), 327-341 (2009)

8. Betten, A., Brinkmann, G., Pisanski, T.: Counting symmetric configurations $v_{3}$. Discrete Appl. Math. 99(1-3), 331-338 (2000). Proceedings of the 5th Twente Workshop on Graphs and Combinatorial Optimization (Enschede, 1997)

9. Boben, M., Pisanski, T.: Polycyclic configurations. Eur. J. Comb. 24(4), 431-457 (2003)

10. Bokowski, J., Schewe, L.: There are no realizable $15_{4}$ - and $16_{4}$-configurations. Rev. Roum. Math. Pures Appl. 50(5-6), 483-493 (2005)

11. Bokowski, J., Grünbaum, B., Schewe, L.: Topological configurations $\left(n_{4}\right)$ exist for all $n \geq 17$. Eur. J. Comb. 30(8), 1778-1785 (2009)

12. Grünbaum, B.: Configurations of points and lines. In: The Coxeter Legacy, pp. 179-225. Amer. Math. Soc., Providence (2006)

13. Grünbaum, B.: A catalogue of simplicial arrangements in the real projective plane. Ars Math. Contemp. 2(1), 1-25 (2009)

14. Grünbaum, B.: Configurations of Points and Lines. Graduate Studies in Mathematics, vol. 103. Amer. Math. Soc., Providence (2009)

15. Grünbaum, B., Rigby, J.F.: The real configuration (214). J. Lond. Math. Soc. (2) 41(2), 336-346 (1990) 